This item was submitted to Loughborough's Research Repository by the author.

Items in Figshare are protected by copyright, with all rights reserved, unless otherwise indicated.

\title{
Deposition of cupric oxide thin films by spin coating
}

PLEASE CITE THE PUBLISHED VERSION

http://dx.doi.org/10.1179/1433075X14Y.0000000204

\section{PUBLISHER}

(c) W.S. Maney \& Son

\section{VERSION}

AM (Accepted Manuscript)

\section{PUBLISHER STATEMENT}

This work is made available according to the conditions of the Creative Commons Attribution-NonCommercialNoDerivatives 4.0 International (CC BY-NC-ND 4.0) licence. Full details of this licence are available at: https://creativecommons.org/licenses/by-nc-nd/4.0/

\section{LICENCE}

CC BY-NC-ND 4.0

\section{REPOSITORY RECORD}

Isherwood, Patrick J.M., Ali Abbas, Jake W. Bowers, Benjamin A. Grew, and Michael Walls. 2019. "Deposition of Cupric Oxide Thin Films by Spin Coating". figshare. https://hdl.handle.net/2134/25435. 


\section{DEPOSITION OF CUPRIC OXIDE THIN FILMS BY SPRIN COATING}

C 2014 Taylor and Francis Group (formerly Maney Publishing). This article may be downloaded for personal use only. Any other use requires prior permission of the authors and the Taylor and Francis Group.

The following article appeared in Materials Research Innovations volume 18 issue 2 (special issue:

Energy Materials), and may be found at http://dx.doi.org/10.1179/1433075X14Y.0000000204 


\title{
Deposition of Cupric Oxide Thin Films by Spin Coating
}

\author{
P. J. M. Isherwood, A. Abbas, J. W. Bowers, B. Grew and J M Walls \\ CREST, School of Electronic, Electrical and Systems Engineering, Loughborough University, Loughborough, Leicestershire, \\ LE11 3TU, UK
}

*Corresponding author: P.J.M.Isherwood@lboro.ac.uk

\begin{abstract}
Cupric oxide thin films were deposited onto soda-lime glass by the spin coating and subsequent annealing of copper nitrate dissolved in a glycerol-water solvent. It was found that the solution consistently gave reproducible films with good adhesion on glass. A range of band gaps were estimated between 0.8 and $1.17 \mathrm{eV}$, showing that this material has potential as a photoabsorber. Resistivity was successfully reduced from $1.47 \times 10^{5} \Omega . \mathrm{cm}$ to $7.02 \Omega . \mathrm{cm}$ by doping the films with sodium. Dopant concentrations of 1 at. wt. \% gave the lowest resistivity, showing that the ideal doping is $1 \%$ or less. Film structure was found to improve with an increase in annealing time from 10 minutes to 1 hour, although this did not have any noticeable effect on either the electrical or optical properties of the films.
\end{abstract}

\section{List of Figures}

Figure 1: Transmission curves for undoped and 5 and $20 \%$ doped spin coated films and an undoped sputtered film for comparison

Figure 2: Estimated band gap against doping concentration

Figure 3: Log-normal graph of resistivity against dopant concentration for both 10 minute and 1 hour anneal times

Figure 4: SEM photomicrographs showing spin coated films with $3 \%$ doping (a) and $20 \%$ doping (b)

Figure 5: SEM photomicrographs of spin coated films with $3 \%$ doping, annealed for 1 hour (a) and 10 minutes (b)

Figure 6: SEM photomicrographs of spin coated films with $3 \%$ doping, annealed for 1 hour (a) and undoped, annealed for 10 minutes (b)

Figure 7: SEM photomicrographs showing a spin coated film with $1 \%$ doping and 1 hour annealing time (a) and a sputtered film (b) for comparison

\section{Introduction}

Over the last decade or so there has been an increasing interest in the use of photovoltaics (PV) for electricity generation. At present, the vast majority of photovoltaic cells produced commercially are made from silicon ${ }^{1}$. Although currently a more expensive power generation technology than more traditional fossil fuel techniques, the cost of electricity produced using PV is reducing rapidly ${ }^{1}$. Ultimately, for PV to be a competitive technology it needs to be cheaper in terms of cost per kilowatthour (kWh) of electricity generated than fossil fuel sources. Although the cost of silicon production is decreasing, material costs will be important in determining the final price, and hence competitiveness, of $\mathrm{PV}$ as an energy generation technology. Whilst silicon is not a rare material, extracting it from silica $\left(\mathrm{SiO}_{2}\right)$ is an energy intensive process ${ }^{2}$, which means that even when made in large quantities it is likely to be relatively expensive. It is therefore important to investigate cheaper alternatives as candidates for PV cells. 
Cupric oxide $(\mathrm{CuO})$ is one of two principal oxides of copper. It is a naturally $\mathrm{p}$-type semiconductor with a near-ideal band gap for solar photovoltaic applications ${ }^{3}$. Despite this there have been relatively few studies on its use as a photovoltaic material, with most groups focussing instead on cuprous oxide $\left(\mathrm{Cu}_{2} \mathrm{O}\right)$. Copper oxides are also a key component of the more successful p-type transparent conductive oxides $^{4-6}$ (TCOs) due to the ability of copper ions to delocalise holes from oxide sites ${ }^{6,7}$. Therefore in order to gain a better understanding of the conduction mechanisms and to look for ways of increasing the conductivity of p-type TCOs, characterisation of the copper oxides is important. Films for use in these areas can be deposited by a range of different processes. Most groups working on copper oxides have used reactive sputtering from a copper metal target $\left(\mathrm{eg},{ }^{8}\right)$. However, this does not readily allow for film doping, and is a relatively expensive process.

This work describes a process for the deposition of cupric oxide by spin coating from a chemical solution and provides an optical, electrical and structural analysis of films deposited by this method. A comparison is made with films deposited by sputter deposition. These data show that this material has potential as a cheap, readily processable alternative to other thin-film photoabsorbers. The method described also allows for the incorporation of variable concentrations of a range of dopants.

\section{Experimental}

Films were deposited by spin coating solutions of copper nitrate dissolved in a glycerol/water solvent. Precursor solutions were formed by dissolving $0.5 \mathrm{~g}$ copper nitrate hexahydrate $(99.99 \%$, Sigma Aldrich) in a solution of $1 \mathrm{ml}$ glycerol and $1 \mathrm{ml}$ de-ionised water. For sodium-doped films, sodium acetate $(99.99 \%$, Sigma Aldrich) was added to the precursor solution. Dopant concentrations were calculated as a percentage of atomic weight, and films were doped with 1, 2, 3, 5, and $20 \%$ sodium. Films were formed by spin-coating these solutions on $50 \mathrm{~mm}$ by $50 \mathrm{~mm}$ precleaned soda-lime glass slides for 90 seconds at $1000-2000 \mathrm{rpm}$. Samples were then dried at $220^{\circ} \mathrm{C}$ in air and subsequently annealed at $450^{\circ} \mathrm{C}$ in air under a fume hood to oxidise the nitrate films. Anneal times were initially kept to 10 minutes. A second set of films containing 1, 3, 5 and $20 \%$ sodium were annealed for 60 minutes. Film characterisation included transmission, Hall mobility, sheet resistance, film thickness measurements and SEM. Material band gaps were calculated from the transmission. Transmission measurements were taken using a Varian Cary 5000 spectrophotometer and covered the spectrum from 2000 to $200 \mathrm{~nm}$. Measurements were taken every $10 \mathrm{~nm}$. Hall measurements were taken using an Ecopia HMS 3000 Hall Mobility system. Thickness measurements were carried out using an Ambios XP2 stylus profilometer.

Sputtered films were deposited from a single stoichiometric ceramic target using an AJA International Orion 8HV sputter coater equipped with an AJA International 600 Series radio frequency power supply. Deposition pressure was kept at 1 millitorr, with an argon flow rate of 7 standard cubic centimetres per minute (SCCM) and a 1\% oxygen in argon flow rate of 1 SCCM. Power was kept at 120 watts. Films were deposited at room temperature and deposition time was 2 hours.

\section{Discussion}

It was found that the spin coating method described consistently produced good films of a range of thicknesses up to around $500 \mathrm{~nm}$. Film thickness was determined by the spin rate, with thicker films forming at slower speeds. Thicker films were found to be significantly less homogenous. This is most likely due to non-linear evaporation of the solvent during the drying step. Because of this variation, doping experiments were run using a spin rate of $2000 \mathrm{rpm}$, which gave films of a thickness of around $120-150 \mathrm{~nm}$ as measured by stylus profilometer. 
Films show high transmission in the infra-red, with a cut-off starting around 800-900 nm. Figure1 shows transmission curves for undoped and doped spin coated films as well as an undoped sputtered film for comparison.

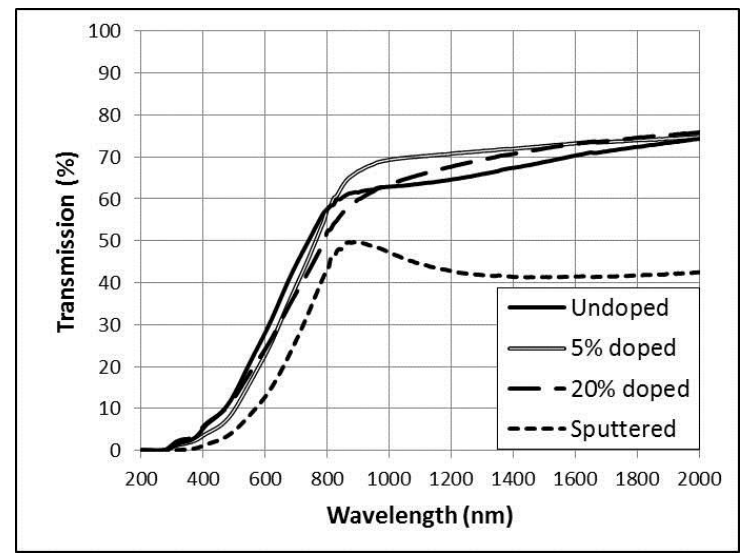

Figure 1: Transmission curves for undoped and 5 and 20\% doped spin coated films and an undoped sputtered film for comparison

None of the films measured showed significant interference fringes. Band gaps were estimated using the absorption coefficient, which was calculated using Equation 1:

1) $\alpha(\mathrm{E})=-\frac{1}{\mathrm{~d}} \ln \left[\mathrm{T}_{\text {normalised }}(\mathrm{E})\right]$

Where $\alpha$ is the absorption coefficient, $d$ is the thickness, $E$ is the photon energy and $T_{\text {normalised }}$ is the normalised transmission for light of that photon energy. They were found to be indirect, and varied from around $0.8 \mathrm{eV}$ to $1.17 \mathrm{eV}$ (Fig. 2), which is slightly lower than previously reported experimental data $^{3}$, but is in good agreement with the calculated theoretical band gap ${ }^{9}$.

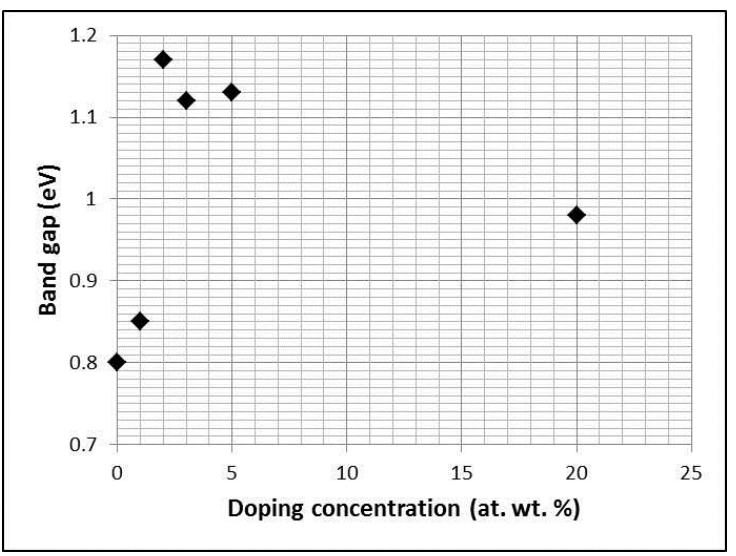

Figure 2: Estimated band gap against doping concentration

Film resistivities were found to vary from highly insulating for undoped films to $7 \Omega . c m$ for $1 \%$ sodium doping and 10 minutes anneal time. Figure 3 shows resistivity against dopant concentration for both 10 minute and 1 hour anneal times. 


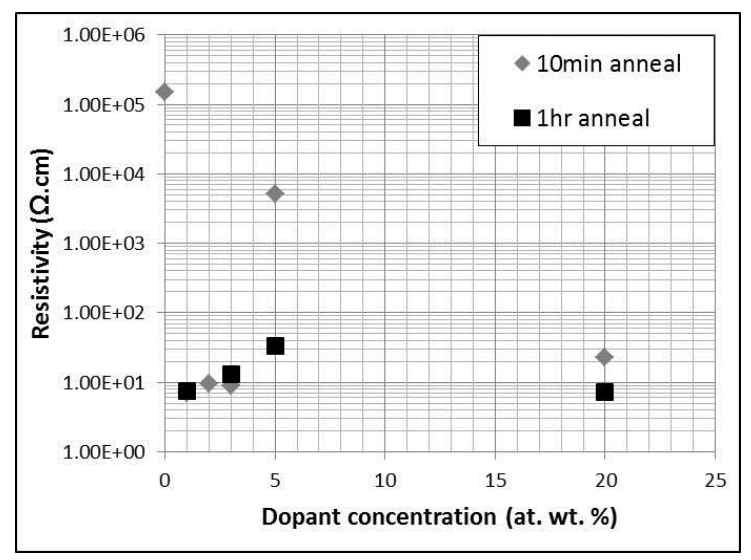

Figure 3: Log-normal graph of resistivity against dopant concentration for both 10 minute and 1 hour anneal times

The reduction in resistivity for even a small dopant concentration is noticeable, with the exception of a doping level of $5 \%$ and 10 minute anneal time. The general trend is for resistivity to increase with doping concentrations above $1 \%$. The exception to this is for films doped with $20 \%$ sodium. This suggests that the ideal sodium dopant level is around $1 \%$ or less, and that higher levels cause increasing amounts of crystal distortion. For a doping level of $20 \%$, it would normally be expected that the crystal structure would be so distorted that the film would be highly insulating, however when compared with films with a much lower dopant concentration using SEM (Fig. 4) there is no apparent change in crystallinity. The resistivity was similar to that of less highly doped films. It is possible that for dopant concentrations this high the cupric oxide structure rejects the majority of the dopant, with only a small proportion being incorporated into the individual grains. The rest is then either burnt off along with the acetate group, or is pushed into the grain boundaries. This type of behaviour has been observed in other materials $\left(\mathrm{eg},{ }^{10,11}\right)$. For concentrations of 1 and $3 \%$, increasing annealing time to one hour didn't appear to alter the resistivity significantly, implying that for lower concentrations the dopant is more readily accepted into the crystal structure.
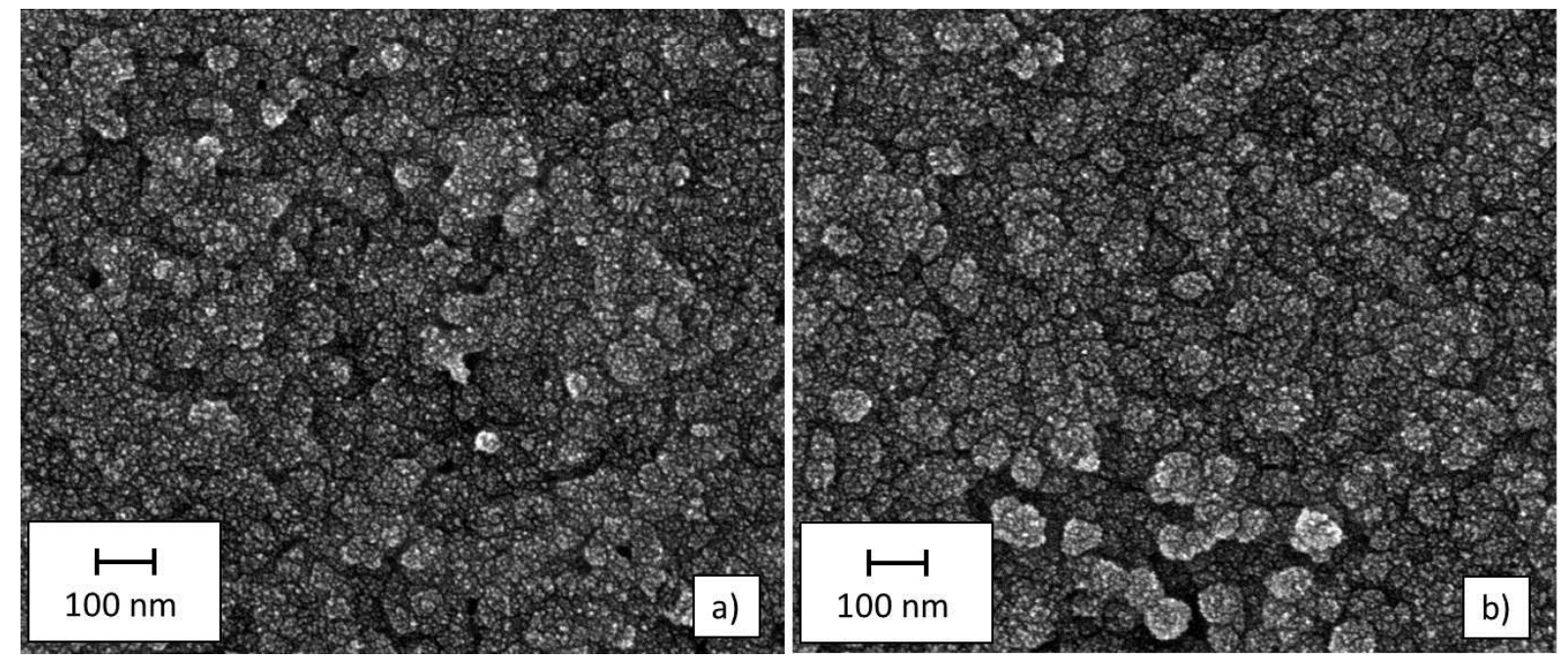

Figure 4: SEM photomicrographs showing spin coated films with $3 \%$ doping (a) and $20 \%$ doping (b)

Increasing the anneal time had a significant impact on film density and structure. SEM images show that films annealed for 10 minutes contain more gaps and holes than those annealed for 1 hour (Fig. 5). Crystal sizes for both sets of films are very similar, and are typically arranged in large rounded clusters. This is significantly more pronounced for films annealed for 1 hour, with the clusters typically becoming more regular in both size and shape (Fig. 5). The only major change in crystallinity is between the undoped films and the doped films. Undoped films annealed for 10 minutes are 
reasonably compact, and are comparable to the doped films after annealing for 1 hour although the individual crystals are smaller (Fig. 6). Dopant concentration does not obviously impact on film growth, with more highly doped films not showing any greater degree of disruption than those with lower dopant concentrations. However it is possible that the changes are too difficult to see at this level, and would require the use of a TEM to observe.
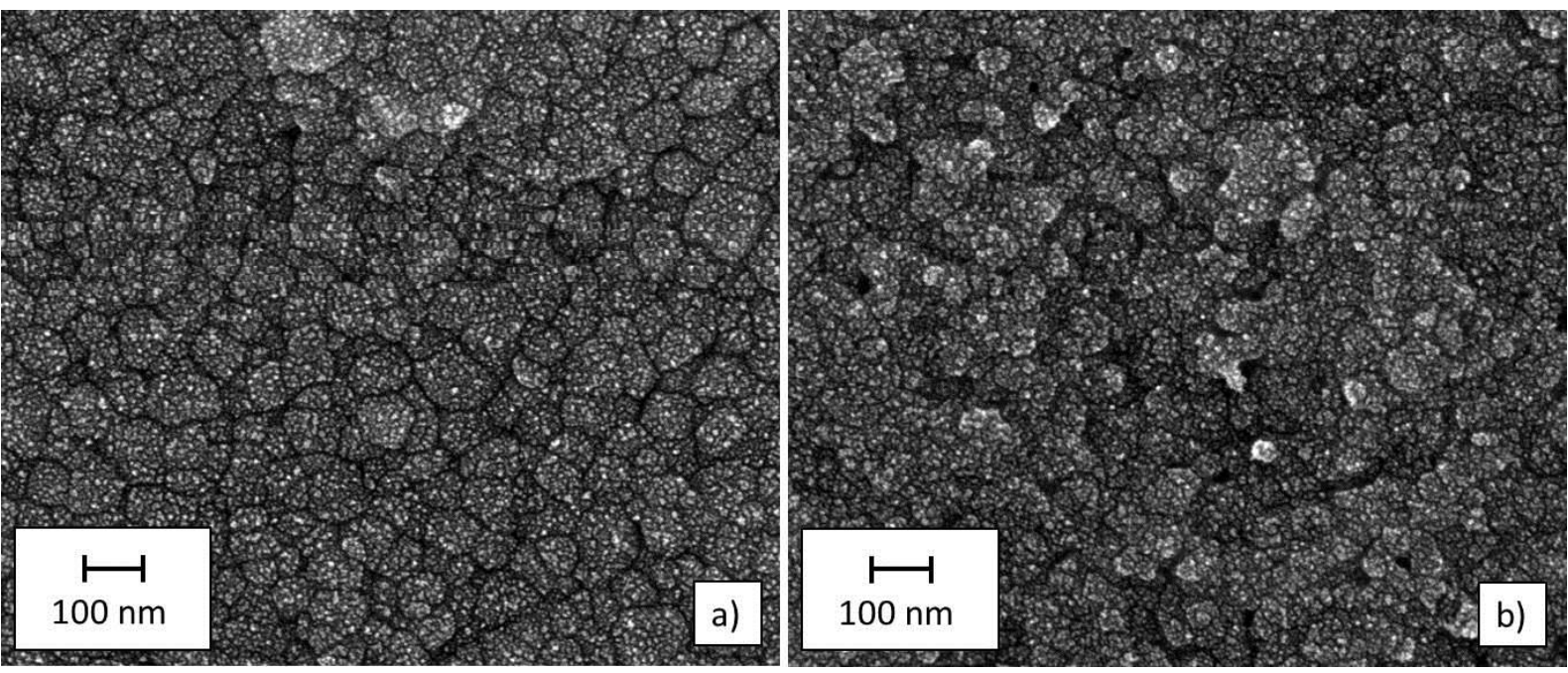

Figure 5: SEM photomicrographs of spin coated films with 3\% doping, annealed for 1 hour (a) and 10 minutes (b)
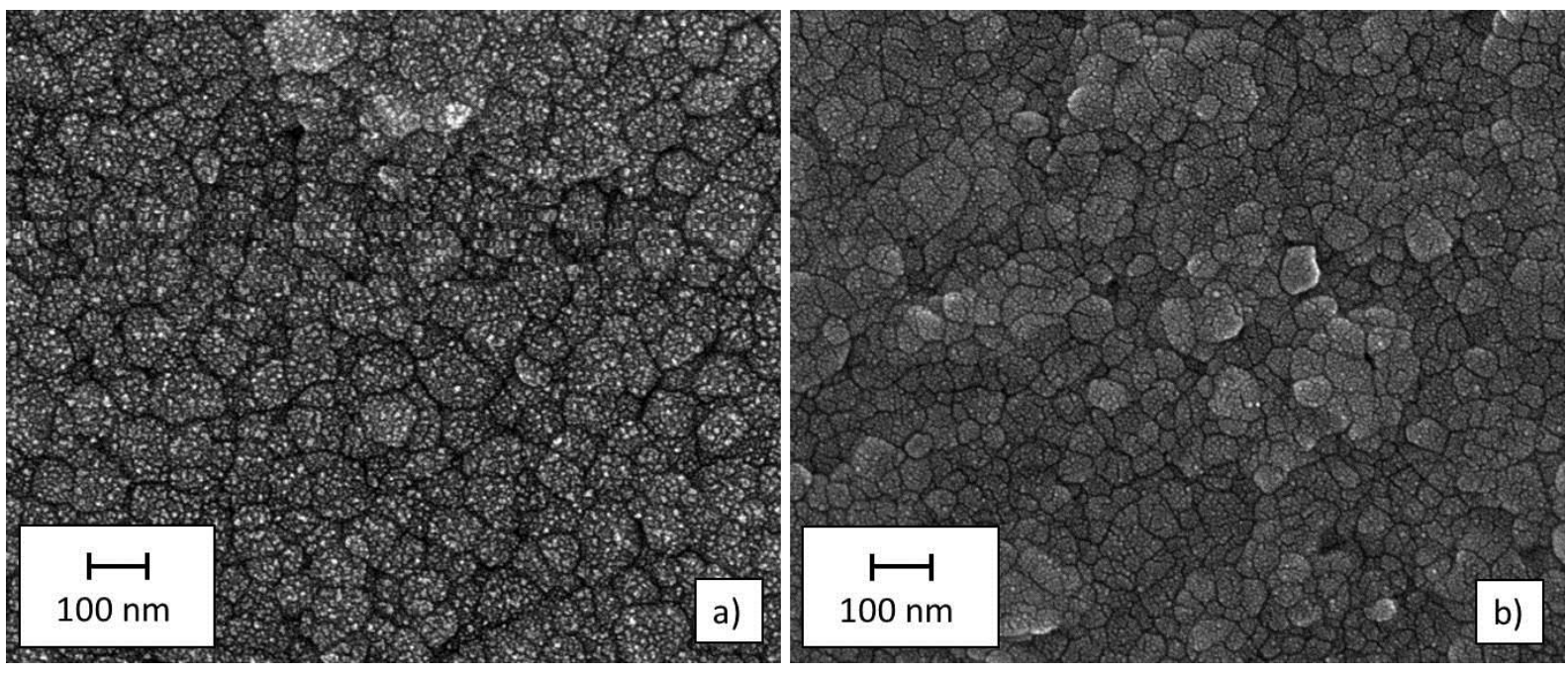

Figure 6: SEM photomicrographs of spin coated films with $3 \%$ doping, annealed for 1 hour (a) and undoped, annealed for 10 minutes (b)

It was found that the optical properties of the spin coated films were largely the same as those of the sputtered films. Sputtered film band gaps were estimated to be between 0.8 and $0.9 \mathrm{eV}$, which is within the range found for the spin coated films. Film thicknesses were similar, with sputtered films being between 150 and $160 \mathrm{~nm}$.

Sputtered films were found to have resistivities of around $1.5 \times 10^{3} \Omega . \mathrm{cm}$, which is two orders of magnitude lower than undoped spin coated films. The reason for this is unknown. Because $\mathrm{CuO}$ is a p-type oxide, depositing the film in a more oxygen-rich environment should increase the conductivity as more copper vacancies are formed. The spin coated films, being deposited in air, were formed in the presence of a much higher oxygen partial pressure than the sputter coated films. It is possible that the evaporation of the solvents and removal of nitrate from the films during the heating and annealing steps reduced the amount of oxygen in the film sufficiently to counteract the effects of the increased oxygen in the deposition atmosphere. Although undoped spin-coated films showed significantly higher 
resistivities, doped films showed resistivities that were up to three orders of magnitude lower than those of the sputtered films. This suggests that with the incorporation of the right amount of sodium dopant, cupric oxide films deposited using non-vacuum techniques can be as good as or better than sputtered films in terms of electrical properties.

Structurally, sputtered and spin coated films were found to be quite different. Sputtered films appear very smooth and dense, with few if any gaps or pinholes. The crystals are much larger, being around $50 \mathrm{~nm}$ in size, as opposed to around $20 \mathrm{~nm}$ for the doped and $10 \mathrm{~nm}$ for the undoped spin coated films. The crystal shapes are also slightly different, with the spin coated films showing small blocky crystals. Those in the sputtered films are more plate like and slightly elongated (Fig. 7).
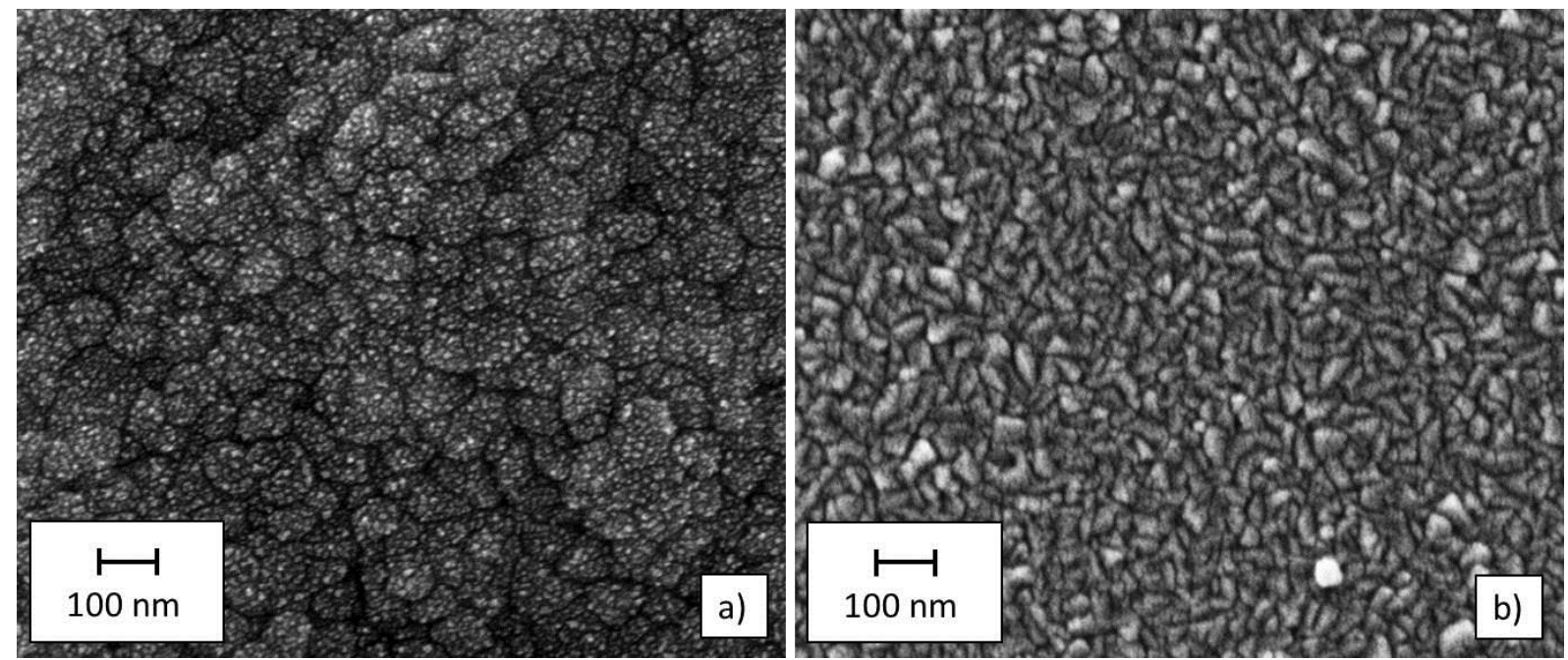

Figure 7: SEM photomicrographs showing a spin coated film with $1 \%$ doping and 1 hour annealing time (a) and a sputtered film (b) for comparison

\section{Conclusions}

A reliable low cost method for producing cupric oxide thin films has been demonstrated. Films produced using this method have been successfully doped with sodium using a range of dopant concentrations. This has been shown to reduce film resistivity from $1.47 \times 10^{5} \Omega . \mathrm{cm}$ to $7.02 \Omega . \mathrm{cm}$, with dopant concentrations of 1 at. wt. \% giving the lowest resistivity. Increasing post-formation annealing time was found to improve film quality without having any apparent effect on the optical or electrical properties. Optical properties of spin coated films were found to be comparable to those of films sputtered in a relatively low oxygen environment. Although undoped spin coated films showed inferior electrical properties to those of sputtered films, doping the films was found to improve them significantly. The best doped spin-coated films showed resistivities three orders of magnitude less than that showed by the sputtered films. Based on SEM images sputtered films were found to be of higher structural quality.

\section{References}

[1] S. S. Hegedus and A. Luque, Eds., "Handbook of Photovoltaic Science and Engineering". Chichester: Wiley and Sons Ltd, 2003, p. 1117.

[2] B. P. Lee, H. M. Lee, D. H. Park, J. S. Shin, T. U. Yu, and B. M. Moon, "Refining of MG-Si by hybrid melting using steam plasma and EMC," Sol. Energy Mater. Sol. Cells, vol. 95, no. 1, pp. 56-58, Jan. 2011. 
[3] L. Wang, K. Han, G. Song, X. Yang, and M. Tao, "Characterization of electro-deposited CuO as a low-cost material for high-efficiency solar cells," Conference Record of the 2006 IEEE 4th World Conference on Photovoltaic Energy Conversion, vol. 1, pp. 130-133, May 2006.

[4] J. Tate, H. Ju, J. Moon, a. Zakutayev, a. Richard, J. Russell, and D. Mclntyre, "Origin of p-type conduction in single-crystal CuAIO2," Phys. Rev. B, vol. 80, no. 16, pp. 1-8, Oct. 2009.

[5] Y. M. Lu, Y. B. He, B. Yang, a. Polity, N. Volbers, C. Neumann, D. Hasselkamp, and B. K. Meyer, "RF reactive sputter deposition and characterization of transparent CuAIO2 thin films," Phys. Status Solidi, vol. 3, no. 8, pp. 2895-2898, Sep. 2006.

[6] H. Yanagi, S. Inoue, K. Ueda, H. Kawazoe, H. Hosono, and N. Hamada, "Electronic structure and optoelectronic properties of transparent p-type conducting CuAlO2," J. Appl. Phys., vol. 88, no. 7, pp. 4159-4163, 2000.

[7] H. Kawazoe, H. Yanagi, K. Ueda, and H. Hosono, "Transparent p-type conducting oxides: design and fabrication of pn heterojunctions," MRS Bull., vol. 25, no. 08, pp. 28-36, 2000.

[8] J. F. Pierson, A. Thobor-Keck, and A. Billard, "Cuprite, paramelaconite and tenorite films deposited by reactive magnetron sputtering," Appl. Surf. Sci., vol. 210, no. 3-4, pp. 359-367, Apr. 2003.

[9] D. Wu, Q. Zhang, and M. Tao, "LSDA+U study of cupric oxide: Electronic structure and native point defects," Phys. Rev. B, vol. 73, no. 23, pp. 1-6, Jun. 2006.

[10] Y. Shigesato, S. Takaki, and T. Haranoh, "Electrical and structural properties of low resistivity tin-doped indium oxide films," J. Appl. Phys., vol. 71, no. 7, p. 3356, 1992.

[11] C. Y. Wong, C. R. M. Grovenor, P. E. Batson, and D. a. Smith, "Effect of arsenic segregation on the electrical properties of grain boundaries in polycrystalline silicon," J. Appl. Phys., vol. 57, no. 2, p. 438, 1985. 